\title{
Association between difficulty initiating sleep in older adults and the combination of leisure-time physical activity and consumption of milk and milk products: a cross-sectional study
}


and Tomohiro Okura ${ }^{5}$

\begin{abstract}
Background: Research has shown that engaging in leisure-time physical activity (LTPA) and consuming dairy foods can lead to better sleep. Combining these two non-invasive prescriptions may be more effective for helping people fall asleep. This study investigates whether participating in LTPA in conjunction with consuming milk and milk products has a beneficial association with difficulty initiating sleep (DIS) among older adults.

Methods: The present study looked at 421 community-dwelling older people aged 65 years and older living in Ibaraki prefecture, Japan (mean age $74.9 \pm 5.5$ years, male 43.7\%). We measured LTPA and sleep latency with the Physical Activity Scale for the Elderly and the Pittsburgh Sleep Quality Index, respectively. Participants who needed 30 minutes or more to fall asleep were defined as having DIS. We assessed dairy consumption as participants' habitual intake of milk, yogurt and cheese.
\end{abstract}

Results: After adjusting for covariates, participants who engaged in sufficient levels of LTPA as well as consumed milk $(\mathrm{OR}=0.27,95 \% \mathrm{Cl}=0.10-0.73)$ or cheese $(\mathrm{OR}=0.34,95 \% \mathrm{Cl}=0.14-0.85)$ were less likely to complain of DIS compared with people who neither engaged in LTPA nor ingested milk or cheese.

Conclusions: Our findings suggest that the combination of engaging in LTPA and consuming milk or cheese is necessary as a prescription to improve falling asleep for older adults suffering from DIS. Additionally, engaging in LTPA along with dairy consumption may effectively improve a problem with falling asleep.

Keywords: Exercise, Dairy, Insomnia, Elderly

\section{Background}

As people age, the quality and quantity of their sleep often deteriorate, and sleep complaints are extremely common in older adults. In a previous study approximately one out of three Japanese older adults reported insomnia symptoms [1]. Difficulty initiating sleep (DIS), difficulty remaining asleep, waking up early in the morning and lack of satisfying sleep are known as insomnia

\footnotetext{
* Correspondence: n.kitano0911@gmail.com

'Doctoral Program in Physical Education, Health and Sport Sciences,

Graduate School of Comprehensive Human Sciences, University of Tsukuba,

1-1-1 Tennodai, Tsukuba, Ibaraki 305-8574, Japan

${ }^{2}$ Research Fellow of the Japan Society for the Promotion of Science, 5-3-1

Kojimachi, Chiyoda-ku, Tokyo 102-0083, Japan

Full list of author information is available at the end of the article
}

symptoms [2], with DIS being one of the most common symptoms in older people. Chronic DIS leads to high risks of mortality [3], diabetes [4], depression [5], poor physical function [6] and cognitive impairment [7]. Finding ways for older adults to improve their ability to fall asleep is essential to their well-being.

It has been established that regular leisure-time physical activity (LTPA), including exercise, has a beneficial effect on a person's physical and psychological health $[8,9]$. Recently, habitual LTPA is being recognized as a potentially effective factor for treating or preventing sleep problems; it is considered a non-pharmacological intervention to improve sleep. Previous randomized control trials (RCTs) $[10,11]$ have revealed that participating 
in LTPA decreases sleep latency (the time required to fall asleep). Furthermore, an epidemiological study [12] showed a relationship between engaging in exercise and a lower prevalence of DIS in older adults.

Primarily in western countries, people have consumed milk and milk products before bedtime as an effective means of improving their sleep. In fact, dairy products contain nutrients, particularly, tryptophan, a precursor to serotonin and melatonin, which play an important role in promoting sleep, including falling asleep [13]. Therefore, ingesting milk and milk products has been considered helpful in improving sleep, and a previous $\mathrm{RCT}$ reported that sleep efficiency is increased by melatonin-rich nighttime milk consumption in older people [14]. Since engaging in LTPA and consuming milk and dairy products are beneficial agents for sleep promotion, combining these two non-invasive prescriptions may more effectively help older adults suffering from DIS. However, there is limited literature on the relationship between sleep in older adults and the combination of these two daily activities. This study has two objectives: confirm independent associations between DIS and engaging in LTPA or consuming milk and milk products, and to examine whether combining these two habits is more strongly associated with a lower prevalence of DIS.

\section{Methods}

\section{Data collection}

A total of 437 community-dwelling, older Japanese individuals participated in the survey. The participants, aged 65 years and older, were recruited in Tsuchiura and Kasama Cities in Ibaraki prefecture, Japan in 2013. The Tsuchiura City participants consisted of 160 community dwellers (mean age $75.6 \pm 6.2$ years, male $34.0 \%$ ) recruited through local advertisements and flyers or invited by municipal employees from June to November 2013. For Kasama City, we used data from 277 randomly selected individuals (mean age $74.5 \pm 5.0$ years, male $49.6 \%$ ) who participated in the "Kasama study" [15] from July to August 2013. We started with 437 participants, but we rejected 16 individuals due to incomplete data. There were 421 final participants (mean age $74.9 \pm 5.5$ years, male $43.7 \%$ ) in the data analysis. All participants provided a signed informed consent. The Ethics committee of the University of Tsukuba approved this study.

\section{Leisure-time physical activity}

To assess the amount of LTPA, we used the Japanese version of the Physical Activity Scale for the Elderly (PASE) [16]. A previous study deemed the PASE to be reliable and valid [16]. LTPA contains five activities: walking (for both recreation and transportation); light-, moderate-, and vigorous-intensity recreational activities; and muscle strength training. We measured a participant's frequency (day/week) and duration (minute/day) of each activity during the previous 7 days and calculated a total LTPA score using frequency, duration and weight for each activity used in the analysis.

\section{Milk/milk products consumption}

We assessed the consumption of milk, yogurt and cheese during the previous couple months using a self-reported questionnaire and the following questions: "How many days a week do you drink milk (or eat yogurt/cheese) [day (s)/week]?" and "How much milk (or yogurt/cheese) do you usually consume each day [milliliters or grams/day]?" Additionally, we supplied examples of portion size (e.g. a glass of milk $=200 \mathrm{ml}$ ) to help participants answer the questions. Using these two questions, we calculated consumption of each food item, and the sum of the three items equaled a participant's total dairy consumption used in the analysis. To estimate total dairy consumption, units of milk intake were converted from milliliters to grams using the following equivalency: $100 \mathrm{ml}=103.2 \mathrm{gm}$ " [17]. No participants had a milk allergy. Previous studies have used self-reported questionnaires to evaluate the frequency and the portion size of daily milk and milk product consumption; those authors confirmed a moderate correlation $(r=0.4-0.6)$ with the weighed dietary recording considered the gold standard for assessing dietary methods $[18,19]$.

\section{Sleep}

We gathered information on total sleep time and sleep latency during the previous month and evaluated these data by referencing the Pittsburgh Sleep Quality Index (PSQI) [20]. PSQI is used to identify sleep disorders in both clinical and research fields throughout the world, and validity and reliability have been confirmed [20].

\section{Potential confounders}

We referenced previous studies to determine minimum required potential confounders for our study: age, gender, body mass index (BMI), hypnotic use for sleep [21], engaging in LTPA [22] and dairy consumption [23,24]. We obtained age, gender and hypnotic use with a selfreport questionnaire. We measured height and weight with a stadiometer or a digital weight scale and calculated BMI calculated as weight in $\mathrm{kg}$ divided by height in meters squared.

\section{Statistical analysis}

LTPA participation was divided into 3 groups based on median level of engagement or no engagement at all: 1) nothing, 2) low level and 3) high level. Total dairy consumption was divided into 3 groups using tertiles: 1) low level, 2) moderate level and 3) high level. A variable composed of the following 4 groups combining 
LTPA and intake of dairy products was created: 1) LTPA with large dairy intake, 2) LTPA with small dairy intake, 3) no LTPA with large dairy intake and 4) no LTPA with small dairy intake. By referencing previous studies [25], people who took more than $30 \mathrm{~min}$ to fall asleep were defined as having DIS.

We analyzed group differences (non-DIS vs. DIS), using independent $t$-test and chi-square test. We used logistic regression analysis to examine the association between participation in LTPA, consumption of dairy products or a combination of those two variables (dependent variables) with DIS (independent variable) adjusting for age, gender, hypnotic use, BMI and those LTPAs or dairy products which were not used as dependent variables. In addition to the odds ratios, we also calculated $P$ for trend. Statistical analysis was performed by IBM SPSS Statistics version 19.0 with the level of significance set at $P<0.05$.

\section{Results}

\section{Description of the study participants}

The characteristics of study participants are shown in Table 1 . The mean age was $74.9 \pm 5.5$ years, and the subjects' usual sleep time was $405.9 \pm 73.4 \mathrm{~min} /$ night. Out of 421 participants, 135 individuals met the definition of DIS (32.1\%) and 80 people (19.0\%) habitually used sleeping pills. Compared with participants who did not have DIS, individuals defined as having DIS were more likely to be women, experienced shorter total sleep duration and long sleep latency, engaged less in LTPA and were more likely to use hypnotics $(P<0.05)$.

\section{LTPA and DIS}

An association between LTPA and DIS is presented in Table 2. After adjusting for age, gender, hypnotic use, BMI and consumption of milk and milk products, a high

Table 1 Characteristics of study participants

\begin{tabular}{|c|c|c|c|}
\hline & All $(n=421)$ & Non-DIS $(n=286)$ & DIS $(n=135)$ \\
\hline & Mean \pm SD & Mean \pm SD & Mean \pm SD \\
\hline Age, years & $74.9 \pm 5.5$ & $74.7 \pm 5.5$ & $75.3 \pm 5.6$ \\
\hline Women, n (\%) & $237(56.3)$ & $149(52.1)$ & $88(65.2)^{*}$ \\
\hline Height, cm & $155.2 \pm 9.0$ & $156.0 \pm 8.6$ & $153.6 \pm 9.5^{*}$ \\
\hline Weight, kg & $56.1 \pm 9.6$ & $56.9 \pm 9.7$ & $54.4 \pm 9.3^{*}$ \\
\hline Body mass index, $\mathrm{kg} / \mathrm{m}^{2}$ & $23.2 \pm 2.9$ & $23.3 \pm 2.9$ & $23.0 \pm 2.8$ \\
\hline Total sleep time, min & $405.9 \pm 73.4$ & $411.6 \pm 70.8$ & $393.7 \pm 77.3^{*}$ \\
\hline Sleep latency, min & $21.1 \pm 21.5$ & $10.7 \pm 5.5$ & $43.2 \pm 25.8^{*}$ \\
\hline Hypnotic use, n (\%) & $80(19.0)$ & $34(11.9)$ & $46^{*}$ \\
\hline Leisure-time physical activity, points & $21.7 \pm 25.1$ & $23.7 \pm 26.9$ & $17.6 \pm 20.5^{*}$ \\
\hline \multicolumn{4}{|l|}{ Milk consumption } \\
\hline Frequency, days/week & $3.6 \pm 2.9$ & $3.8 \pm 3.0$ & $3.2 \pm 2.9$ \\
\hline Volume, ml/day & $155.9 \pm 127.1$ & $162.9 \pm 129.4$ & $141.0 \pm 121.1$ \\
\hline Total consumption, ml/week & $784.3 \pm 813.4$ & $819.0 \pm 833.1$ & $710.9 \pm 767.7$ \\
\hline \multicolumn{4}{|l|}{ Yogurt consumption } \\
\hline Frequency, days/week & $4.0 \pm 2.8$ & $3.9 \pm 2.9$ & $4.1 \pm 2.8$ \\
\hline Volume, g/day & $104.6 \pm 79.0$ & $100.6 \pm 72.2$ & $113.4 \pm 91.9$ \\
\hline Total consumption, g/week & $506.6 \pm 491.4$ & $469.9 \pm 418.7$ & $587.1 \pm 616.0^{*}$ \\
\hline \multicolumn{4}{|l|}{ Cheese consumption } \\
\hline Frequency, days/week & $1.3 \pm 1.8$ & $1.3 \pm 1.8$ & $1.3 \pm 2.0$ \\
\hline Volume, g/day & $14.3 \pm 16.6$ & $15.6 \pm 17.3$ & $11.5 \pm 14.8^{*}$ \\
\hline Total consumption, g/week & $35.6 \pm 55.3$ & $37.4 \pm 56.6$ & $31.9 \pm 52.6$ \\
\hline \multicolumn{4}{|l|}{ Total dairy consumption } \\
\hline Frequency, days/week & $5.4 \pm 2.4$ & $5.4 \pm 2.4$ & $5.3 \pm 2.3$ \\
\hline Volume, g/day & $193.9 \pm 149.4$ & $194.2 \pm 145.0$ & $193.5 \pm 159.0$ \\
\hline Total consumption, g/week & $1357.5 \pm 1045.9$ & $1359.1 \pm 1015.0$ & $1354.3 \pm 1112.9$ \\
\hline
\end{tabular}

*P< 0.05 (Non-DIS vs. DIS).

DIS: difficulty initiating sleep (30 $\mathrm{min} \leq$ sleep latency).

SD: standard deviation. 
Table 2 Association between LTPA and DIS

\begin{tabular}{|c|c|c|c|}
\hline & \multicolumn{3}{|l|}{ DIS } \\
\hline & $\mathrm{n}(\%)$ & Odds ratio & $95 \% \mathrm{Cl}$ \\
\hline Leisure-time physical activity & & \multicolumn{2}{|c|}{ Trend $P$ value $=0.076$} \\
\hline Not engaging & $57(14.4)$ & 1.00 & \\
\hline Low level (0.1-16.9 points) & $168(42.3)$ & 0.72 & $0.38-1.37$ \\
\hline High level ( $\geq 17.0$ points) & $172(43.3)$ & 0.48 & $0.25-0.93$ \\
\hline
\end{tabular}

Bold numbers indicate $P<0.05$.

DIS: difficulty initiating sleep (30 $\mathrm{min} \geq$ sleep latency); LTPA: leisure-time physical activity; $95 \% \mathrm{Cl}$ : $95 \%$ confidence interval.

Odds ratios and $95 \%$ confidence intervals were adjusted for age, gender, hypnotic use, body mass index and consumption of milk and milk products.

level of LTPA was associated with decreased prevalence of DIS compared to no engagement in LTPA (OR $=0.48$, 95\% CI $=0.25-0.93$ )

\section{Milk/milk products and DIS}

As shown in Table 3, participants were less likely to perceive DIS when they consumed a high level of milk $(\mathrm{OR}=0.47,95 \% \mathrm{CI}=0.26-0.84)$ even after adjusting for age, gender, hypnotic use, BMI, LTPA engagement and yogurt and cheese intake. Moreover, a significant doseresponse relationship was observed between milk intake and prevalence of DIS (Trend $P<0.05$ ). There were no significant associations between consumption of yogurt, cheese or total dairy and DIS.

\section{LTPA with milk/milk products and DIS}

As Table 4 indicates, after controlling for age, gender, hypnotic use, BMI and yogurt and cheese consumption, participants who engaged in LTPA as well as consumed milk were less likely to complain of DIS compared with those who neither engaged in LTPA nor consumed milk (OR $=0.27,95 \% \mathrm{CI}=0.10-0.73)$. Similarly, after adjusting for multiple confounding variables, we found that the combination of LTPA engagement and cheese consumption was associated with decreased prevalence of DIS $(\mathrm{OR}=0.34,95 \% \mathrm{CI}=0.14-0.85)$. We did not find a significant association between DIS and the combination of LTPA and yogurt or total dairy consumption.

\section{Discussion}

We found that higher levels of LTPA and greater milk consumption were each associated with lower prevalence of DIS in older adults. Furthermore, we found that combining LTPA participation and milk and/or cheese consumption was more favorably linked with lower prevalence of DIS.

\section{Description of the study participants}

In the present study, complaints of DIS were reported by $37.1 \%$ of women and by $25.5 \%$ of men, which is similar to other Japanese studies [1]. Furthermore, since a large body of evidence also indicates there is a higher prevalence of insomnia in older women than in older men

Table 3 Association between milk and milk products consumption and DIS

\begin{tabular}{|c|c|c|c|c|}
\hline & \multicolumn{4}{|l|}{ DIS } \\
\hline & $n(\%)$ & Odds ratio & $95 \% \mathrm{Cl}$ & \\
\hline Milk consumption & & Trend $P$ value $=0.041$ & & \\
\hline Not consuming & $105(26.4)$ & 1.00 & & \\
\hline Low level (0-1049 ml/w) & $149(37.5)$ & 0.66 & $0.37-1.18$ & \\
\hline High level ( $\geq 1050 \mathrm{ml} / \mathrm{w}$ ) & $143(36.0)$ & 0.47 & $0.26-0.84$ & \\
\hline Yogurt consumption & & Trend $P$ value $=0.554$ & & \\
\hline Not consuming & $72(18.1)$ & 1.00 & & \\
\hline Low level (0-599 g/w) & $164(41.3)$ & 0.98 & $0.51-1.87$ & \\
\hline High level ( $\geq 600 \mathrm{~g} / \mathrm{w})$ & $161(40.6)$ & 1.27 & $0.66-2.45$ & \\
\hline Cheese consumption & & Trend $P$ value $=0.089$ & & \\
\hline Not consuming & $179(45.1)$ & 1.00 & & \\
\hline Low level (0-49 g/w) & $115(29.0)$ & 0.58 & $0.33-1.01$ & \\
\hline High level ( $\geq 50 \mathrm{~g} / \mathrm{w})$ & $103(25.9)$ & 0.61 & $0.34-1.08$ & \\
\hline Total dairy consumption & & Trend $P$ value $=0.242$ & & \\
\hline Low level $(0-805 \mathrm{~g} / \mathrm{w})$ & $133(33.5)$ & 1.00 & & \\
\hline Moderate level (806-1605 g/w) & $132(33.2)$ & 1.22 & 0.71 & 2.10 \\
\hline High level ( $\geq 1606 \mathrm{~g} / \mathrm{w})$ & $132(33.2)$ & 1.22 & 0.43 & 1.34 \\
\hline
\end{tabular}

Bold numbers indicate $P<0.05$.

DIS: difficulty initiating sleep (30 min $\geq$ sleep latency); $95 \% \mathrm{Cl}: 95 \%$ confidence interval.

Odds ratios and $95 \%$ confidence intervals were adjusted for age, gender, hypnotic use, body mass index, leisure-time physical activity and consumption of milk and milk products that were not used as independent variables. 
Table 4 Associations between DIS and LTPA along with milk and milk products consumption

\begin{tabular}{|c|c|c|c|c|c|}
\hline & & & \multicolumn{3}{|l|}{ DIS } \\
\hline & & & $\mathrm{n}(\%)$ & Odds ratio & $95 \% \mathrm{Cl}$ \\
\hline LTPA & & Milk & & & \\
\hline Not engaging & $x$ & Not consuming & $19(4.8)$ & 1.00 & \\
\hline Not engaging & $x$ & Consuming & $38(9.6)$ & 0.42 & $0.13-1.37$ \\
\hline Engaging & $x$ & Not consuming & $86(21.7)$ & 0.45 & $0.16-1.30$ \\
\hline Engaging & $\times$ & Consuming & $254(64.0)$ & 0.27 & $0.10-0.73$ \\
\hline LTPA & & Yogurt & & & \\
\hline Not engaging & $\times$ & Not consuming & $14(3.5)$ & 1.00 & \\
\hline Not engaging & $\times$ & Consuming & $43(10.8)$ & 1.08 & $0.30-3.96$ \\
\hline Engaging & $\times$ & Not consuming & $58(14.6)$ & 0.57 & $0.16-2.06$ \\
\hline Engaging & $\times$ & Consuming & $282(71.0)$ & 0.64 & $0.20-2.03$ \\
\hline LTPA & & Cheese & & & \\
\hline Not engaging & $\times$ & Not consuming & $26(6.5)$ & 1.00 & \\
\hline Not engaging & $x$ & Consuming & $31(7.8)$ & 0.66 & $0.21-2.09$ \\
\hline Engaging & $x$ & Not consuming & $153(38.5)$ & 0.59 & $0.24-1.45$ \\
\hline Engaging & $x$ & Consuming & $187(47.1)$ & 0.34 & $0.14-0.85$ \\
\hline LTPA & & Total dairy & & & \\
\hline Not engaging & $x$ & Low level (0-12933 g/w) & $32(8.1)$ & 1.00 & \\
\hline Not engaging & $\times$ & High level ( $\geq 12934 \mathrm{~g} / \mathrm{w}$ ) & $25(6.3)$ & 1.90 & $0.62-5.82$ \\
\hline Engaging & $\times$ & Low level (0-12933 g/w) & $167(42.1)$ & 0.83 & $0.36-1.91$ \\
\hline Engaging & $x$ & High level ( $\geq 12934 \mathrm{~g} / \mathrm{w})$ & $173(43.6)$ & 0.77 & $0.33-1.76$ \\
\hline
\end{tabular}

Bold numbers indicate $P<0.05$

DIS: difficulty initiating sleep (30 min $\geq$ sleep latency); LTPA: leisure-time physical activity.

Odds ratios and $95 \%$ confidence intervals were adjusted for age, gender, hypnotic use, body mass index and consumption of milk and milk products that were not used as independent variables.

[26], the rates of insomnia in the study participants do not appear to be specific to our study population.

\section{LTPA and DIS}

In this study, sufficient LTPA was associated with a lower prevalence of DIS, which is in accord with a large amount of research showing that exercise improves sleep latency $[10,11,27]$ and physically fit individuals have shorter sleep latency [28]. Although the mechanisms are not clear, earlier studies have suggested that physical activity may improve sleep due to 1) body temperature increases, 2) energy expenditure, 3) antianxiety and antidepressant effects and 4) entrainment of circadian rhythm $[22,29]$. The improvement we saw in DIS in our study may be a consequence of these physiological and psychological effects of LTPA.

\section{Milk/milk products and DIS}

We found a positive link between decreased prevalence of DIS and the intake of milk, but not yogurt, cheese or total dairy consumption. At present, there are divergent opinions as to the effects of milk on sleep; some researchers found that consuming milk improves sleep quality [30,31], whereas, others reported no significant effects of milk intake [14,32]. Yamamura et al. demonstrated a significant improvement in sleep efficiency after ingesting melatonin-rich milk before bedtime [14]. From results of our study, we suggest that daily intake of ordinary commercial milk may also facilitate falling asleep. Our findings confirmed the beneficial effect that commercial milk consumption can have on the prevalence of DIS, which is useful information for the older adult population.

We also found a significant linear trend between drinking milk and a lower prevalence of DIS, that is, DIS became less frequent as the amount of milk consumed increased. To our knowledge, literature concerning the association between sleep and milk/milk products intake in older people is limited. In particular, the dose-response relationship between these factors is still unclear. Hence, our findings contribute to this developing area of research.

\section{LTPA with milk/milk products and DIS}

To our knowledge, although some studies have investigated the relationship between sleep and either LTPA or milk/milk products, no prior study has evaluated the association between DIS and LTPA in conjunction with dairy intake. Our research produced interesting and novel 
findings as it shows that older adults who engaged in LTPA and consumed milk or cheese reported DIS less frequently than people who neither engaged in LTPA nor consumed milk or cheese. Additionally, individuals who only engaged in LTPA or only consumed milk or cheese had higher odds ratios than people with both habits. The combination of LTPA and milk or cheese consumption may be an effective tool to help people fall asleep.

In modern society, older adults frequently complain of insomnia symptoms including difficulty falling asleep [1]. They may turn to medication to attain sleep, although medications have a greater risk of side effects e.g. falling [33] and even death [34]. Attele et al. showed that older people are often reluctant to take sleeping pills because of the negative impacts on their health [35], hence, non-pharmacological therapy with less adverse effects is needed. Along with the beneficial effects on sleep, LTPA provides other health benefits such as maintaining physical function [36] and decreased risks of mortality [37] and dementia [38]. Milk and milk products also contribute significant amounts of calcium, which plays an important role in reducing risks of osteoporosis [39] and hypertension [40]. Due to these benefits, participating in LTPA and consuming milk or dairy foods are recommended for well-being in later life. Furthermore, taking into account our findings on sleep improvement, we suggest that habitual LTPA along with milk or cheese consumption are helpful not only for maintaining general health, but also as a non-drug intervention for DIS in older adults.

\section{Limitations}

There are some limitations in our study. First, the generalization of the study's results is uncertain. Almost all study participants entered the study after receiving an invitation letter or seeing a local advertisement. Thus, the study may underestimate the proportion of older adults with poor living habits (e.g. inactive, low consumption of dairy products or insomnia) or those in poor health. Additionally, sample size was small, specifically with regards to people who did not participate in LTPA and/or consume dairy products; this limits confidence in the results. To verify our results, future investigations with larger sample sizes in unhealthy, older adults are needed. Second, we did not assess depressive symptoms, cognitive function/dementia, chronic illness (e.g. heart disease or lung disease) [41,42] or a number of medications [43] which could be potential confounders for our results. Third, since LTPA, dairy consumption and sleep were assessed via self-reported questionnaires, there may be recalling/reporting bias in our data. In particular, Silva et al. suggested that sleep latency might be overestimated by a self-reported measure [44], thus, future studies should include objective measures of sleep, such as the use of actigraphy. Fourth, because of the present study's cross-sectional design, we could not prove a causal relationship. There may be a bidirectional relationship between physical activity and sleep $[45,46]$. To reveal a causal relationship, a prospective cohort study and an intervention study are needed. Finally, unfortunately, we did not assess the times of day that participants' practiced LTPA and consumed milk/dairy products. The effect that exercise has on initiating sleep differs depending on when it was performed [22]. Similarly, ingesting milk and other milk products, which contain several nutrients with potential sleep-promoting properties, may be effective for falling asleep when these food items are consumed before bedtime.

Because earlier studies have revealed that LTPA and dairy intake separately have positive effects on DIS, it is reasonable to conclude from our findings that LTPA in combination with milk or cheese intake may help decrease DIS, even with the above study limitations.

\section{Conclusions}

We studied the association between LTPA in conjunction with milk or other dairy products intake and DIS in older adults. Our data revealed that higher levels of LTPA related to a lower prevalence of DIS and decreased complaints of difficulty falling asleep, in a dose-response relationship, with consumption of milk. Moreover, it is worth mentioning that people who engage in LTPA and drink milk or eat cheese may be falling asleep easily in contrast to those who do not engage in either of those activities. This information could be useful in the development of a non-pharmacological intervention for insomnia and to help promote sleep quality in older people.

\section{Abbreviations}

DIS: Difficulty Initiating Sleep; LTPA: Leisure-time Physical Activity; BMI: Body Mass Index; PSQI: Pittsburgh Sleep Quality Index.

\section{Competing interests}

The authors declare that they have no competing interests.

\section{Authors' contributions}

$\mathrm{KN}$. planned and designed the study, performed the statistical analysis and drafted the manuscript. KN, TK, TT, OY. and JT. collected and interpreted the data. TK and OT. supervised and coordinated the study. TK, TT, OY, JT, TK and OT. developed the manuscript. All authors read and approved the final manuscript.

\section{Acknowledgements}

This study was supported by the Ministry of Education, Culture, Sports, Science and Technology of Japan, Grant-in-Aid for Scientific Research (B), 25282213, 2013-2016.

\section{Author details}

${ }^{1}$ Doctoral Program in Physical Education, Health and Sport Sciences, Graduate School of Comprehensive Human Sciences, University of Tsukuba, 1-1-1 Tennodai, Tsukuba, Ibaraki 305-8574, Japan. ²Research Fellow of the Japan Society for the Promotion of Science, 5-3-1 Kojimachi, Chiyoda-ku, Tokyo 102-0083, Japan. ${ }^{3}$ Physical Fitness Research Institute, Meiji Yasuda Life Foundation of Health and Welfare, 150 Tobuki, Hachioji, Tokyo 192-0001, Japan. ${ }^{4}$ Gerontology Research Center, University of Jyväskylä, PO Box 35, 
Jyväskylä 40014, Finland. ${ }^{5}$ Faculty of Health and Sport Sciences, University of Tsukuba, 1-1-1 Tennodai, Tsukuba, Ibaraki 305-8574, Japan.

Received: 11 May 2014 Accepted: 4 November 2014 Published: 18 November 2014

\section{References}

1. Kim K, Uchiyama M, Okawa M, Liu X, Ogihara R: An epidemiological study of insomnia among the Japanese general population. Sleep 2000, 23:41-47.

2. Ancoli-Israel S, Poceta JS, Stepnowsky C, Martin J, Gehrman P: Identification and treatment of sleep problems in the elderly. Sleep Med Rev 1997, 1:3-17.

3. Kripke DF: Sleep and mortality. Psychosom Med 2003, 65:74

4. Kawakami N, Takatsuka N, Shimizu H: Sleep disturbance and onset of type 2 diabetes. Diabetes Care 2004, 27:282-283.

5. Kaneita Y, Ohida T, Uchiyama M, Takemura S, Kawahara K, Yokoyama E Miyake T, Harano S, Suzuki K, Fujita T: The relationship between depression and sleep disturbances: a Japanese nationwide general population survey. J Clin Psychiatry 2006, 67:196-203.

6. Dam T-TL, Ewing S, Ancoli-Israel S, Ensrud K, Redline S, Stone K: Association between sleep and physical function in older men: the osteoporotic fractures in men sleep study. J Am Geriatr Soc 2008, 56:1665-1673.

7. Blackwell T, Yaffe K, Ancoli-Israel S, Schneider JL, Cauley JA, Hillier TA, Fink $\mathrm{HA}$, Stone $\mathrm{KL}$ : Poor sleep is associated with impaired cognitive function in older women: the study of osteoporotic fractures. J Gerontol Ser A Biol Sci Med Sci 2006, 61:405-410

8. Buchman AS, Boyle PA, Wilson RS, Bienias JL, Bennett DA: Physical activity and motor decline in older persons. Muscle Nerve 2007, 35:354-362.

9. Blake $H$, Mo P, Malik S, Thomas S: How effective are physical activity interventions for alleviating depressive symptoms in older people? A systematic review. Clin Rehabil 2009, 23:873-887.

10. King AC, Oman RF, Brassington GS, Bliwise DL, Haskell WL: Moderate-intensity exercise and self-rated quality of sleep in older adults. A randomized controlled trial. J Am Med Assoc 1997, 277:32-37.

11. Li F, Fisher KJ, Harmer P, Irbe D, Tearse RG, Weimer C: Tai chi and self-rated quality of sleep and daytime sleepiness in older adults: a randomized controlled trial. J Am Geriatr Soc 2004, 52:892-900.

12. Inoue $\mathrm{S}$, Yorifuji T, Sugiyama M, Ohta T, Ishikawa-Takata K, Doi H: Does habitual physical activity prevent insomnia? A cross-sectional and longitudinal study of elderly Japanese. J Aging Phys Act 2013, 21:119-139.

13. Peuhkuri K, Sihvola N, Korpela R: Diet promotes sleep duration and quality. Nutr Res 2012, 32:309-319.

14. Yamamura S, Morishima H, Kumano-go T, Suganuma N, Matsumoto $H_{\text {, }}$ Adachi H, Sigedo Y, Mikami A, Kai T, Masuyama A, Takano T, Sugita Y, Takeda M: The effect of Lactobacillus helveticus fermented milk on sleep and health perception in elderly subjects. Eur J Clin Nutr 2009, 63:100-105.

15. Tsunoda K, Tsuji T, Kitano N, Mitsuishi Y, Yoon J-Y, Yoon J, Okura T: Associations of physical activity with neighborhood environments and transportation modes in older Japanese adults. Prev Med 2012, 55:113-118.

16. Hagiwara A, Ito N, Sawai K, Kazuma K: Validity and reliability of the Physical Activity Scale for the Elderly (PASE) in Japanese elderly people. Geriatr Gerontol Int 2008, 8:143-151.

17. The Ministry of Science and Technology: 5 th revision of the standard tables of food composition in Japan. 2000. Available from URL: http:// www.mext.go.jp/b_menu/shingi/gijyutu/gijyutu3/toushin/05031802/002/ 013.pdf (in Japanese)

18. Michaëlsson $\mathrm{K}$, Melhus $\mathrm{H}$, Bellocco R, Wolk A: Dietary calcium and vitamin D intake in relation to osteoporotic fracture risk. Bone 2003, 32:694-703.

19. Ozawa M, Ohara T, Ninomiya T, Hata J, Yoshida D, Mukai N, Nagata M, Uchida K, Shirota T, Kitazono T, Kiyohara Y: Milk and dairy consumption and risk of dementia in an elderly Japanese population: the Hisayama Study. J Am Geriatr Soc 2014, 62:1224-1230.

20. Doi Y, Minowa M, Okawa M, Uchiyama M: Development of the Japanese version of the Pittsburgh Sleep Quality Index. Jpn J Psychiatry Treat 1998 13:755-763 (in Japanese)

21. Glass J, Lanctôt KL, Herrmann N, Sproule BA, Busto UE: Sedative hypnotics in older people with insomnia: meta-analysis of risks and benefits. BMJ 2005, 331:1169.

22. Driver HS, Taylor SR: Exercise and sleep. Sleep Med Rev 2000, 4:387-402.

23. Cronin FJ, Krebs-Smith SM, Wyse BW, Light L: Characterizing food usage by demographic variables. J Am Diet Assoc 1982, 81:661-673.
24. Ministry of Health, Labour and Welfare: National Health and Nutrition Survey. ; 2012. Available from URL: http://www.mhlw.go.jp/bunya/kenkou/eiyou/dl/ h24-houkoku-04.pdf (in Japanese).

25. Guilleminault C, Clerk A, Black J, Labanowski M, Pelayo R, Claman D: Nondrug treatment trials in psychophysiologic insomnia. Arch Intern Med 1995, 155:838-844.

26. Foley DJ, Monjan AA, Brown SL, Simonsick EM, Wallace RB, Blazer DG: Sleep complaints among elderly persons: an epidemiologic study of three communities. Sleep 1995, 18:425-432.

27. King AC, Pruitt LA, Woo S, Castro CM, Ahn DK, Vitiello MV, Woodward SH, Bliwise DL: Effects of moderate-intensity exercise on polysomnographic and subjective sleep quality in older adults with mild to moderate sleep complaints. J Gerontol A Biol Sci Med Sci 2008, 63:997-1004.

28. Edinger JD, Morey MC, Sullivan RJ, Higginbotham MB, Marsh GR, Dailey DS, McCall WV: Aerobic fitness, acute exercise and sleep in older men. Sleep 1993, 16:351-359.

29. Youngstedt SD: Effects of exercise on sleep. Clin Sports Med 2005 24:355-365. xi

30. Brezinová V, Oswald I: Sleep after a bedtime beverage. BMJ 1972, 2:431-433.

31. Southwell PR, Evans CR, Hunt JN: Effect of a hot milk drink on movements during sleep. BMJ 1972, 2:429-431.

32. Valtonen M, Niskanen $L$, Kangas A-P, Koskinen T: Effect of melatonin-rich night-time milk on sleep and activity in elderly institutionalized subjects. Nord J Psychiatry 2005, 59:217-221.

33. Ensrud KE, Blackwell TL, Mangione CM, Bowman PJ, Whooley MA, Bauer DC, Schwartz AV, Hanlon JT, Nevitt MC: Central nervous system-active medications and risk for falls in older women. J Am Geriatr Soc 2002, 50:1629-1637.

34. Kripke DF, Klauber MR, Wingard DL, Fell RL, Assmus JD, Garfinkel L: Mortality hazard associated with prescription hypnotics. Biol Psychiatry 1998, 43:687-693.

35. Attele AS, Xie JT, Yuan CS. Treatment of insomnia: an alternative approach. Altern Med Rev 2000, 5:249-259.

36. Keysor J: Does late-life physical activity or exercise prevent or minimize disablement? A critical review of the scientific evidence. Am J Prev Med 2003, 25:129-136.

37. Stessman J, Hammerman-Rozenberg R, Cohen A, Ein-Mor E, Jacobs JM: Physical activity, function, and longevity among the very old. Arch Intern Med 2009, 169:1476-1483.

38. Rovio S, Kåreholt I, Helkala E-L, Viitanen M, Winblad B, Tuomilehto J, Soininen $H$, Nissinen A, Kivipelto M: Leisure-time physical activity at midlife and the risk of dementia and Alzheimer's disease. Lancet Neurol 2005, 4:705-711.

39. Nieves JW: Osteoporosis: the role of micronutrients. Am J Clin Nutr 2005, 81:1232S-1239S

40. Engberink MF, Hendriksen MAH, Schouten EG, van Rooij FJA, Hofman A, Witteman JCM, Geleijnse JM: Inverse association between dairy intake and hypertension: the Rotterdam Study. Am J Clin Nutr 2009, 89:1877-1883.

41. Foley D, Ancoli-Israel S, Britz P, Walsh J: Sleep disturbances and chronic disease in older adults: results of the 2003 National Sleep Foundation Sleep in America Survey. J Psychosom Res 2004, 56:497-502.

42. Burton LC, Shapiro S, German PS: Determinants of physical activity initiation and maintenance among community-dwelling older persons. Prev Med 1999, 29:422-430

43. Ancoli-Israel S, Cooke JR: Prevalence and comorbidity of insomnia and effect on functioning in elderly populations. J Am Geriatr Soc 2005, 53: S264-S271.

44. Silva GE, Goodwin JL, Sherrill DL, Arnold JL, Bootzin RR, Smith T, Walsleben JA, Baldwin CM, Quan SF: Relationship between reported and measured sleep times: the sleep heart health study (SHHS). J Clin Sleep Med 2007, 3:622-630.

45. Holfeld B, Ruthig JC: A longitudinal examination of sleep quality and physical activity in older adults. J Appl Gerontol 2014, 33:791-807.

46. Dzierzewski JM, Buman MP, Giacobbi PR, Roberts BL, Aiken-Morgan AT, Marsiske M, McCrae CS: Exercise and sleep in community-dwelling older adults: evidence for a reciprocal relationship. J Sleep Res 2014, 23:61-68.

doi:10.1186/1471-2318-14-118

Cite this article as: Kitano et al:: Association between difficulty initiating sleep in older adults and the combination of leisure-time physical activity and consumption of milk and milk products: a cross-sectional study. BMC Geriatrics 2014 14:118. 